\title{
Anti-human ovarian cancer, cytotoxicity, and antioxidant effects of Nigella Sativa green-formulated Au nanoparticles: Describing a new chemotherapeutic supplement
}

\author{
Type \\ Research paper
}

\section{Keywords}

cytotoxicity, antioxidant, Gold nanoparticles, Nigella Sativa seeds, anti-human ovarian cancer

\begin{abstract}
Introduction

The recent research showed that the Gold nanoparticles (GNPs) formulated with Nigella Sativa aqueous extract having potent antioxidant and anti-human ovarian cancer activities in vitro condition.

Material and methods

For determinate the properties of the GNPs that were produced from the reaction between gold chloride solution with aqueous Nigella Sativa extract, we used UV-Visible Spectroscopy (UV-Vis), Field Emission Scanning Electron Microscopy (FE $₫$ SEM), Fourier Transformed Infrared Spectroscopy (FT $\square$ IR), and Transmission Electron Microscopy (TEM). For evaluating anti-ovarian cancer and cytotoxicity effects of GNPs, Au chloride, and Nigella Sativa aqueous extract, we used MTT assay.
\end{abstract}

\section{Results}

The result of this test showed that GNPs have no cytotoxicity on normal cell line (HUVEC) and have potent anti-ovarian cancer features dose-dependently against PA-1, SK-OV-3, and SW-626 cell lines. The IC50 of GNPs were 249, 361, and $433 \mu \mathrm{g} / \mathrm{mL}$ against PA-1, SW-626, and SK-OV-3 cell lines, respectively. For evaluating the antioxidant features of GNPs, Au chloride, and Nigella Sativa aqueous extract, we used the DPPH test, in this test butylated hydroxytoluene was a positive control, the results of this test showed that the GNPs have an effective antioxidant feature. In the antioxidant test, the IC50 of GNPs and BHT were 144 and $201 \mu \mathrm{g} / \mathrm{mL}$, respectively.

\section{Conclusions}

Probably, potent anti-human ovarian cancer activities of GNPs formulated with Nigella Sativa aqueous seed extract because of antioxidant properties. After evaluating the effectiveness of this formulation in clinical trial researches, it can be a good alternative to chemotherapy drugs. 


\title{
Anti-human ovarian cancer, cytotoxicity, and antioxidant effects of
}

Nigella Sativa green-formulated Au nanoparticles: Describing a new

\author{
chemotherapeutic supplement
}

\author{
Haibo Ruan#, Li Wang²\#, Minyuan Wang1, Weiwei Mo', Jichao Jin¹, \\ Arunachalam Chinnathambi ${ }^{3}$, Tahani Awad Alahmadi ${ }^{4}$, Velmurugan \\ Meganathan $^{5}$, Ling Zhang** \\ ${ }^{1}$ Gynecology, The First People's Hospital of Wenling, Wenling, Zhejiang, 317500, China. \\ ${ }^{2}$ Gynecology, Affiliated Hospital of Shandong University of Traditional Chinese Medicine, Jinan, \\ Shandong, 250014, China. \\ ${ }^{3}$ Department of Botany and Microbiology, College of Science, King Saud University, PO Box - \\ 2455, Riyadh -11451, Saudi Arabia. \\ ${ }^{4}$ Department of Pediatrics, College of Medicine and King Khalid University Hospital, King Saud \\ University, Medical City, PO Box-2925, Riyadh -11461, Saudi Arabia. \\ ${ }^{5}$ Department of Cellular and Molecular Biology, University of Texas Health Science Center at \\ Tyler, Tyler, Texas, United States. \\ ${ }^{6}$ Gynecology, Northwest Women and Children's Hospital, Xi 'an, Shaanxi, 710061, China. \\ * Corresponding author: Ling Zhang: zhangling12347@ sina.com \\ \# HaiBo Ruan and Li Wang are co-first aothors, they contributed equally to this work.
}




\section{ABSTRACT}

This research showed that the Gold nanoparticles (GNPs) formulated with Nigella Sativa aqueous extract having potent antioxidant and anti-human ovarian cancer activities in vitro condition. For determinate the properties of the GNPs that were produced from the reaction between gold chloride solution with aqueous Nigella Sativa extract, we used UV-Visible Spectroscopy (UVVis), Field Emission Scanning Electron Microscopy (FE-SEM), Fourier Transformed Infrared Spectroscopy (FT-IR), and Transmission Electron Microscopy (TEM). For evaluating anti-ovarian cancer and cytotoxicity effects of GNPs, Au chloride, and Nigella Sativa aqueous extract, we used MTT assay. The result of this test showed that GNPs have no cytotoxicity on normal cell line (HUVEC) and have potent anti-ovarian cancer features dose-dependently against PA-1, SK-OV3, and SW-626 cell lines. The IC50 of GNPs were 249, 361, and $433 \mu \mathrm{g} / \mathrm{mL}$ against PA-1, SW626, and SK-OV-3 cell lines, respectively. For evaluating the antioxidant features of GNPs, Au chloride, and Nigella Sativa aqueous extract, we used the DPPH test, in this test butylated hydroxytoluene was a positive control, the results of this test showed that the GNPs have an effective antioxidant feature. In the antioxidant test, the IC50 of GNPs and BHT were 144 and 201 $\mu \mathrm{g} / \mathrm{mL}$, respectively. Probably, potent anti-human ovarian cancer activities of GNPs formulated with Nigella Sativa aqueous seed extract because of antioxidant properties. After evaluating the effectiveness of this formulation in clinical trial researches, it can be a good alternative to chemotherapy drugs.

KEYWORDS Gold nanoparticles; Nigella Sativa seeds; anti-human ovarian cancer; antioxidant; cytotoxicity. 


\section{Introduction}

Nigella Sativa is an ethnomedicinal plant with several pharmacological features. It belongs to the Plantae kingdom, Ranunculales order, Ranunculaceae family, and Nigella genus. Nigella Sativa is an annual plant with delicate flowers that are usually yellow, white, pink, pale blue, or purple with 5 to 10 petals. The height of the plant reaches 20 to $90 \mathrm{~cm}$. Nigella Sativa fruit has a large capsule with numerous seeds in it [1-3]. People have consumed Nigella Sativa to control and treat liver function, gastrointestinal, respiratory, cardiovascular, immune, and urinary systems diseases [4- 12]. The seeds of Nigella Sativa and its products such as seeds tincture and roasted black seeds have been used to treat asthma, diarrhea, rheumatism, bronchitis, dropsy, loss of appetite, treatment of indigestion, dysmenorrhoea, amenorrhoea, treatment of skin eruptions and worms and, antiemetic. [4,13-15]. Chemical composition that isolated from Nigella Sativa are as fallow: thymoquinone, dithymoquinone, t-anethol, thymohydroquinone, dihydroxy-28-methy-lolean-12enoate, p-cymene, 4-terpineol, $\alpha$-pinene, thymol, carvacrol, thymol, sesquiterpene longifolene, nigellicine, nigellicimineN-oxide, nigellicimine, nigellidine, alpha-hederin, saponin, citronellol, carvone, limonene, protein, fat, carbohydrates, fiber, $\mathrm{Cu}, \mathrm{Zn}, \mathrm{Fe}, \mathrm{P}$, carotene, vitamin $\mathrm{A}$, avenasterol-5-ene, oleic acid, dihomolinoleic acid, eicodadienoic acid, $\alpha$-sitosterol, linoleic acid, campesterol, saponin, stigma-5, $\beta$-amyrin, obtusifoliol, $\beta$-amyrin, cycloartenol, aliphatic alcohol,

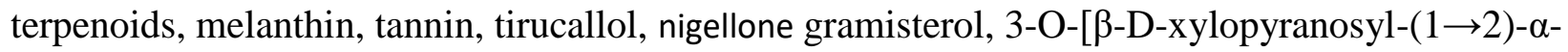
L-rhamnopyranosyl-(1 $\rightarrow 2)-\beta$-D-glucopyranosyl]-11-methoxy-16, citrostadienol , cycloart-23methyl-7, Ns-D1, Ns-D2, etc [16-20]. Researches have shown that Nigella Sativa has many treatment properties such as antibacterial, antifungal, anticonvulsant and, neuro-pharmacological, testicular-protective, anti-asthmatic, pulmonary-protective, nephroprotective, hepato-protective, 
gastro-protective, cardiovascular, immunomodulatory activity, analgesic and anti-inflammatory, anticancer, antidiabetic, antioxidant, and anti-schistosomiasis effects [2, 27-38].

Ovarian cancer is prevalent in all of the world. Predisposing factors for ovarian cancer are as fallow: obesity and overweight, gynecologic surgery, hormone therapy, breast cancer, age, family history, reproductive history, human, talcum powder, and papillomavirus [39,40]. Initially, ovarian cancer developed with the growth of an abnormal cell and then propagation to the whole uterus and all parts of the body [40]. Ovarian cancer has symptoms such as lethargy, weight loss, dyspnea, nausea, pain or pressure in the pelvis, backache, early satiety, abdominal pain, constipation, unexpected vaginal bleeding, urinary frequency, bloating and dyspnea [41]. Diagnosis of ovarian cancer base of these tests blood tests, biopsy, laparoscopy, and imaging examination [42]. A great number of doctors use chemotherapy, immunotherapy, and radiation therapy to treat ovarian cancer [43]. Chemotherapeutic drugs have a bad effect on the body, so today the formulation of an effective chemotherapy drug from GNPs is important $[44,45]$.

Nanotechnology is the field to yield modern systems, tools, and materials by taking control at the atomic and molecular levels using the features that appear on those surfaces. Applications for nanotechnology in medical diagnostics, food, medicine, biotechnology, environment, energy, chemistry, physics, etc, introduce this technology as an interdisciplinary and crosssectoral context. The interdisciplinary nature of nanoscience and nanotechnology as the field to yield modern systems, tools, and materials with precision atoms and molecules, will sooner or later affect the health and medical sector [44,45]. Drug use is currently volumetric, so most cells in the body need medication. In the new method, the drug is directed directly to specific cells with new injection devices and delivered to the required location. By this mechanism, small and large diseases can be diagnosed and treated at the beginning of their formation [44-46]. The 
National Nanotechnology Project is being implemented in European countries, the United States and Japan with high priority in various fields. Nanotechnology and nanoscience emerging fields can move materials very accurately, to understand and control unprecedented fundamental components of physical objects. It seems that these developments will change the way we design and build everything from vaccines to computers. The plan would increase investment in nanotechnology about twice as much each year as last year. A branch of nanotechnology is the formulation of new drugs with metal nanoparticles [45,46]. Today, nanoparticles have become very popular due to their wide applications in biology, medicine and medicine. Structurally, their size is in the range of 100 nanometers. Several drugs such as small hydrophobic and hydrophilic drugs, molecules, and vaccines of biological nanoparticles can be administered by these nanoparticles. They are widely used in improving the treatment and diagnosis of diseases. Nanoparticles in nanoliposomes, carbon nanotubes, nanofibers, nanospheres have been widely used for drug carriers and in the manufacture of cell scaffolds [44-46]. Applications of nanoparticles in drug delivery include drug carriers in diseases such as cancer, cardiovascular disease, and Alzheimer's. The use of these nanocarriers is very effective for neurological diseases such as Alzheimer's. Due to their size, these nanoparticles can cross the blood-brain barrier, which has always been a barrier to the passage of drugs to the affected area in this type of destructive brain disease. Due to their small size, nanoparticles can also be used in brain cancers [46]. The goal in making nanoparticles is to control the surface properties, particle size, and release of a specific and efficient drug in a specific place and time for the drug to be as effective as possible. Nanoparticles are widely used in tissue engineering scaffolds, targeted drug delivery and disease diagnosis. At present, many drug delivery systems are made of nanoparticles and different materials have been used as drug stimulants or enhancers to 
ameliorate the effectiveness of treatment and the durability and stability as well as the safety of anticancer drugs [45]. The substances used to release cancer drugs are divided into different polymers, magnetic, and biomolecules. These materials can also provide surface modifications such as binding to target antibodies and ligands to make the nanoparticles act purposefully to increase the effectiveness of the treatment [44-46].

GNPs as famous metallic nanoparticles with Pharmaceutic properties recently have been used for the treatment of some kind of cancers and tumors [44-46]. The results of a study showed that GNPs have anti-acute myeloid leukemia features in the cellular and molecular state, GNPs widely killed all malignant leukemia cells (32D-FLT3-ITD, Human HL-60/vcr, and Murine C1498) in the nano concentrations [46]. So far, there have been no reports of anti-human ovarian cancer properties of GNPs green-synthesized by herbs. However, several herbal medicines such as Turmeric [Curcuma longa], Quercetin [Quercus tinctoria], Genistein, Mayapple [Podophyllum peltatum], Shatavari [Asparagus racemosus], Camptotheca [Camptotheca acuminate], Ginkgo biloba, Lodhra [Symplocus racemosa], Pacific yew [Taxus brevifolia], Garlic [Allium sativum], Ashoka [Saraca indica], Green tea [Camellia sinensis], and Neem [Azadirachta indica] were used in ancient times to cure ovarian cancer [47]. In the current research, the properties of GNPs formulated by Nigella Sativa aqueous extract against common human ovarian cancer cell lines i.e., SK-OV-3, SW-626, and PA-1 were evaluated.

\section{Material and Methods}

\subsection{Material}


Bovine serum, antimycotic antibiotic solution, 2,2-diphenyl-1- pikrilhydrazil (DPPH), dimethyl sulfoxide (DMSO), decamplmaneh fetal, 4- (Dimethylamino) benzaldehyde, hydrolyzate, Ehrlich solution, and borax-sulfuric acid mixture, Dulbazolic mixture Modified Eagle Medium (DMED), all were afforded from the US Sigma-Aldrich company.

\subsection{Synthesis of GNPs containing Nigella sativa seed aqueous extract}

To produce a green synthesis of GNPs first stage is Extraction from Nigella sativa. The method that we used for achieving this purpose was extracted Nigella sativa seed with distilled water in the microwave. Generally, the method that we used for the synthesis gold nanoparticles containing Nigella sativa seeds extract are as follows in $50 \mathrm{~mL}$ of distilled water we dissolved $1.5 \mathrm{~g}$ of $\mathrm{NaOH}$ pellets and $100 \mathrm{~mL}$ of $\mathrm{HAuCl}_{4} \times \mathrm{H}_{2} \mathrm{O}(1 \mathrm{mM})$ and followed by this act, added $20 \mathrm{~mL}$ of Nigella sativa seeds extract and then stirred for $1 \mathrm{~h}$ at $25^{\circ} \mathrm{C}$. After this process, the solution color changed to the dark yellow color that indicated the formation of GNPs. Finally, the solution was let to precipitate, then the precipitate was filtered and washed with ethanol, acetone, and distilled water. The final precipitate was dried for 14 hours at $90{ }^{\circ} \mathrm{C}$ to provide the best GNPs powder [46].

\subsection{Chemical characterization of GNPs containing Nigella Sativa aqueous extract}

Several techniques were used to indicate the characterizations of GNPs are as follows: TEM, UVVis, and FT-IR. 
The TEM is a method by which the size and shape of nanoparticles were determined.

UV-Vis spectroscopy analysis is a method by which the characteristic absorption bands of gold were evaluated.

FT-IR (Shimadzu IR affinity.1) was used to identify the potential biomolecules in the Nigella Sativa aqueous extract that participate in the reduction of gold nanoparticles.

\subsection{Antioxidant activities of GNPs containing Nigella sativa seed aqueous extract}

The free radical scavenging test was first performed by Blois in 1958, and after some modification by numerous studies in its current form. DPPH method is one of the most widely used methods for estimating antioxidant content. DPPH is a stable radical that reacts with hydrogen atom compounds. This test is based on the inhibition of DPPH, which causes the decolorization of DPPH solution by adding radical species or antioxidants. DPPH changes color from purple to yellow by taking an electron from the antioxidant compound. The free radicals in DPPH are adsorbed at 517 $\mathrm{nm}$, which follows Beer Lambert's law, and decreased absorption is linearly related to the amount of antioxidants; the higher the amount of antioxidants, the more DPPH is consumed and the more purple turns yellow [46].

This experiment was performed with few changes in the method of Lu et al [46]. $0.5 \mathrm{ml}$ of $0.1 \mathrm{mM}$ DPPH solution prepared in $95 \%$ ethanol was mixed with $100 \mu \mathrm{l}$ of Nigella sativa seed aqueous extract, GNPs, and gold salt at the concentrations of the $0-1000 \mu \mathrm{g} / \mathrm{ml}$. The resulting solution was 
kept in the dark at $38^{\circ} \mathrm{C}$ for 31 minutes. The absorbance of the samples was then read at $518 \mathrm{~nm}$ [46].

To compare the activity of Nigella sativa seed aqueous extract, GNPs, and gold salt, standard BHT compound was used as a standard antioxidant [46].

To determine the amount of IC50 (IC50 is defined as the concentration required to inhibit 50\% of the antioxidant activity) for Nigella sativa seed aqueous extract, GNPs, and gold salt, experiments were performed at eleven different concentrations of the desired nanoparticle solution and BHT. Each experiment was performed in three shifts and the mean values were calculated [46].

Percentage of radicalization activity was calculated through the following equation [46]:

$$
\text { Inhibition }(\%)=\frac{\text { Sample A. }}{\text { Control A. }} \times 100
$$

In this regard, the blank adsorption indicates the adsorption of the control solution, which contains $0.5 \mathrm{ml}$ of DMPH solution and $100 \mu \mathrm{l}$ of $95 \%$ ethanol instead of Nigella sativa seed aqueous extract, GNPs, and gold salt solutions and adsorption of the reaction indicates the adsorption of the solution content of the Nigella sativa seed aqueous extract, GNPs, and gold salt samples [46]. 
In this research, we used the following cell lines to evaluating anti-human ovarian cancer and cytotoxicity effects of Nigella sativa seed aqueous extract, GNPs, and gold salt using an MTT method.

a) Human ovarian cancer cell lines: PA-1 (ATCC CRL-1572), SW 626 (ATCC HTB-78), SK-OV-

3 (ATCC HTB-77): A panel of 3 ovarian cancer cell lines with varying degrees of genetic complexity. They have genomic mutations in one or more of the following genes according to the Sanger COSMIC database: APC, CDKN2A, FAM123B, KRAS, MLH1, NRAS, PIK3CA, STK11, and TP53.

b) Normal cell line: HUVEC.

Each cell line was placed separately in T25 flasks with a complete culture medium (including DMEM (Dulbecco's Modified Eagle Medium, 10\% complementary bovine fetal serum, and 1\% penicillin-streptomycin solution) and at $37^{\circ} \mathrm{C}$ in the incubator, cell culture was incubated with $5 \%$ $\mathrm{CO}_{2}$.

After obtaining $80 \%$ cell density, the sample was exposed to $1 \%$ trypsin-EDTA solution and after 3 minutes of incubation at $37^{\circ} \mathrm{C}$ in a cell culture incubator with $5 \% \mathrm{CO}_{2}$ and observation of cells removed from the bottom of the plate, the sample was centrifuged at $5000 \mathrm{rpm}$ for 5 minutes and then the cell precipitate was decrypted by adding trypsin culture medium. Then, the cell suspensions after adding trypan blue dye were counted by neobar slide and cytotoxicity test was performed by MTT method. For this purpose, in each well of 98 cell culture plate, 10000 cells 
were introduced with $200 \mu \mathrm{L}$ from the complete cell culture medium and to achieve the cell monolayer density, the plate was re-exposed to $5 \% \mathrm{CO}_{2}$ at $37^{\circ} \mathrm{C}$. After reaching $80 \%$ cell growth, the culture medium was removed and the cell surface was first washed with PBS buffer, again, in all wells, a complete two-concentration culture medium of $100 \mu 1$ was introduced and $100 \mu 1$ of solutions of Nigella sativa seed aqueous extract, GNPs, and gold salt at the concentrations of the 0-1000 $\mu \mathrm{g} / \mathrm{ml}$ dissolved in PBS was introduced into well No. 1. After mixing the nanoparticles in the culture medium, $100 \mu \mathrm{l}$ of it was removed and added to the second well. In the next step, 100 $\mu l$ of the second well was removed after stirring the medium and added to well 3 . This operation was performed up to well 11 and thus the amount of nanoparticles in each well was halved, respectively. Well No. 12 contained only one cell and complete culture medium of one concentration and remained as a control. The plate was again exposed to $5 \% \mathrm{CO}_{2}$ at $37^{\circ} \mathrm{C}$ for 24 hours and after 24 hours the cytotoxicity was determined using tetrazolium dye. $10 \mu \mathrm{l}$ of tetrazolium dye $(5 \mathrm{mg} / \mathrm{ml})$ was added to all wells, including the control, and the plate was exposed to $5 \% \mathrm{CO}_{2}$ at $37^{\circ} \mathrm{C}$ for 2 hours. The dye was then removed from the wells and $100 \mu$ of DMSO (Dimethyl sulfoxide) was added to the wells, the plate was wrapped in aluminum foil and shaken thoroughly in a shaker for 20 minutes. Finally, cell survival was recorded in ELISA reader at 540 nm [46]:

$$
\text { Cell viability }(\%)=\frac{\text { Sample A. }}{\text { Control A. }} x 100
$$


Then, based on the absorption rate of each well and its comparison with the control, the inhibitory concentration of 50\% (IC50) was obtained [46].

After collecting data, Minitab statistical software was used for statistical analysis. Evaluation of antioxidant results in a completely randomized design and comparison of means was Duncan posthoc test with a maximum error of $5 \%$. To measure the percentage of cell survival in factorial experiments with the original design of completely randomized blocks and compare the means, Duncan post-hoc test with a maximum error of 5\% was used. The 50\% cytotoxicity (IC50) and 50\% free radical scavenging (IC50)) was estimated with ED50 plus software (INER, V: 1.0). Measurements were reported as mean \pm standard deviation.

\subsection{Statistical analysis}

The data were gathered and given into the "SPSS-24" a computer software program and was analyzed by "one-way ANOVA", and then Duncan posthoc test $(p \leq 0.01)$.

\section{Results and Discussion}

Cancer is now one of the leading causes of death worldwide. Existing treatments have not been able to meet the treatment needs for various types of cancer. Therefore, the use of new technologies 
in the prevention and treatment of cancer can be helpful. Extensive research on nanoparticles has been conducted in recent years [44]. The advent of nanotechnology has had a profound effect on many areas of healthcare and scientific research. Common cancer treatments, including chemotherapy, radiation and surgery, may reduce the size of the tumor, but the effect of these methods is transient and has no positive effect on patient survival. Therefore, replacing more effective, more specific therapies with fewer side effects with higher anti-cancer activity is a dominant issue in clinical oncology $[44,45]$.

The gradual maturation of nanotechnology has been considered not only for treating cancer but also for a wide variety of applications, especially for drug delivery and diagnostic and imaging cases. There are many types of nanoparticles available and choosing the right carriers according to demand is a key issue [46]. Nanoparticles are very close in size to biological molecules in terms of size and can easily penetrate into the cell, for this reason, one of the goals of nanotechnology is to mount molecules and drugs on nanoparticles and transfer them to the target cell [44-46]. It is also possible to create different surface properties for nanoparticles by attaching protective ligands to increase the nanoparticles' resistance to the immune system and increase their presence in the bloodstream, and even binding ligands to specifically bind the nanoparticles to the target tissue $[44,46]$. 
In this experiment, we formulated GNPs by using of Nigella sativa seed aqueous extract. furthermore, in vitro condition, we evaluated the anti-human ovarian cancer activities of GNPs against common human ovarian cancer.

\subsection{FE-SEM analysis of GNPs containing Nigella sativa seed aqueous extract}

FE-SEM analysis is a method for assessment of the size and morphology of materials such as GNPs and is extensively utilized in chemistry, physics, and biology investigates. In this study, the FE-SEM image of GNPs containing Nigella sativa seed aqueous extract is shown in Figure 1. In this figure, the GNPs are spherical, 15-20 nm in size that closes together. Few GNPs seem to shape the agglomerated structure, which is caused by the hydroxyl group.

\subsection{TEM analysis of GNPs containing Nigella sativa seed aqueous extract}

TEM is a method for evaluating the size, shape, and distribution of materials. Figure 2 shows that the GNPs containing Nigella sativa seed extract are spherical and about15-20 nm in sizes, furthermore a small number of them can be seen in other forms. 
Figure 3 reveals the UV-Vis spectra analysis from GNPs containing Nigella sativa seed aqueous extract. In this study UV-Vis spectroscopic analysis indicated that an absorption peak at $519 \mathrm{~nm}$, in this peak the formation of GNPS is approved, thanks to some researches that have been done the wavelength range for GNPs formation from pharmaceutic plants have been determined between $510-550 \mathrm{~nm}[46]$.

\subsection{FT-IR spectrum analysis of GNPs containing Nigella sativa seed aqueous extract}

We used the FT-IR spectrum to determine potential biomolecules in the Nigella sativa seed aqueous extract which are responsible for reducing GNPs. Figure 4 shows the FT-IR spectrum of GNPs containing Nigella sativa seed aqueous extract in the wavenumber of $400-4000 \mathrm{~cm}^{-1}$. In figure 5, wavenumber 3396 is due to hydroxyl and phenolic groups. The bands at 2999, 13391602, 1057, and $531 \mathrm{~cm}^{-1}$ are referred to as asymmetric $\mathrm{CH}_{2}$ stretching, aromatic rings, $\mathrm{C}-\mathrm{O}$ stretching, and $\mathrm{Au}-\mathrm{O}$ respectively.

\subsection{Cytotoxicity and anti-ovarian cancer activities of GNPs containing Nigella sativa seed aqueous extract}

One of the cytotoxicity test methods to measure the rate of cell death is the MTT method, which is based on the formation of formazan dye by reducing the substance MTT (dimethyl thiazole 2 
and 5 diphenyltetrazolium bromide) or other tetrazolium salts [46]. By breaking the MTT tetrazolium ring by mitochondrial enzymes in living cells, insoluble purple formazan crystals are formed. The formation of these crystals indicates the activity of respiratory chain enzymes and is a measure of cell viability. By measuring the amount of absorption by spectrophotometer at specific wavelengths, the number of living cells can be determined. This test is performed according to ISO 10993-5 and its purpose is in vitro evaluation of cytotoxicity. Cytotoxicity test is performed according to ISO10993-5 standard and in three ways: NRU test, CFU test, MTT test and XTT test. The most common method for assessing cytotoxicity is to measure cell survival by MTT [46]. The basis of MTT method is based on the intensity of dye produced by the mitochondrial activity of cells, that measured at a wavelength of 540 to $630 \mathrm{~nm}$ and directly proportional to the number of living cells, the increase or decrease in the number of living cells is linearly related to the activity of cell mitochondria. MTT tetrazolium dye is revived in active (metabolically) cells. Mitochondrial dehydrogenases in living cells produce NADH and NADPH, leading to an insoluble purple precipitate called formazan. This precipitate can be dissolved by isopropanol or dimethyl sulfoxide [46]. Dead cells, on the other hand, are unable to perform this conversion due to the inactivity of their mitochondria and therefore do not show a signal. In this method, dye formation is used as a marker for the presence of living cells. In recent years, MTT testing has been the most important measurement method to evaluate the toxicity and anti-cancer effects of metal nanoparticles [46]. 
In this investigation, the treated cells with different concentrations of the present $\mathrm{HAuCl}_{4}, \mathrm{Nigella}$ sativa seed aqueous extract, and GNPs were assessed by MTT assay for $48 \mathrm{~h}$ about the cytotoxicity properties on normal (HUVEC) and ovarian malignancy cell lines i.e. SW-626, SK-OV-3, and PA1. The absorbance rate was evaluated at $570 \mathrm{~nm}$, which represented viability on normal cell line (HUVEC) even up to $1000 \mu \mathrm{g} / \mathrm{mL}$ for $\mathrm{HAuCl}_{4}$, Nigella sativa seed aqueous extract, and GNPs (Figure 5; Table 1). The viability of malignant ovarian cell line reduced dose-dependently in the presence of $\mathrm{HAuCl}_{4}$, Nigella sativa seed aqueous extract, and GNPs. The IC50 of gold nanoparticles (GNPs) were 249, 361, and $433 \mu \mathrm{g} / \mathrm{mL}$ against PA-1, SW-626, and SK-OV-3 cell lines, respectively (Figure 5; Table 1). Metallic nanoparticles have various parameters such as shape, size, texture, etc. Sizes have very significant properties in the therapeutic effects of nanoparticles, some studies reported that small metallic nanoparticles have better entrance into cells and have significant anti-cancer activities. It has been assessed that particle size lower than $50 \mathrm{~nm}$ showed an excellent remedial feature in the corresponding cancer cell lines [49-53]. As you see in Figures 1 and 2 of our study, the average size of GNPs. synthesized by Nigella sativa seed aqueous extract is $17.5 \mathrm{~nm}$. Gold nanoparticles have been utilized to treat various cancers including Lewis lung carcinoma, human glioma, human lung cancer, uterus cancer, lung epithelial cancer, colon cancer, and mammary carcinoma.

\subsection{Antioxidant features of GNPs containing Nigella sativa seed aqueous extract}


Oxidative stress is caused by an imbalance between the production of free radicals and metabolic reactions, which leads to damage to lipids, proteins and nucleic acids. These damages may be due to low levels of antioxidants or an excessive increase in the production of free radicals in the body [54,55]. In humans, oxidative stress is associated with chronic diseases such as diabetes and cancer. Therefore, the production of synthetic and natural antioxidants is necessary to prevent oxidative stress and its destructive effects. Antioxidants effectively and in various ways reduce the harmful effects of free radicals in the biological and food systems and cause detoxification [5456]. In this regard, green nanoparticles can be used (using plant substrates to prepare nanomaterials that are environmentally friendly and do not contain any harmful chemicals) that show antioxidant properties. At present, the use of non-toxic substances in synthesizing nanoparticles to prevent biological hazards, especially in medical and pharmaceutical applications is considered [33-38]. Many researchers have focused on bioactive substances derived from plants or other sources such as bacteria, fungi and yeast for synthesizing nanoparticles. The green synthesis method is thought to increase the biocompatibility and performance of metal nanoparticles for biological applications due to removing harmful chemicals [56,57]. During the bioproduction stages of nanoparticles, their extracellular production using plants or their extracts is more beneficial and their production can be adjusted in a controlled way based on size, distribution and shape for different purposes [54-57]. 
The scavenging capacity of Nigella sativa seed aqueous extract green-synthesized gold nanoparticles and BHT at different concentrations expressed as percentage inhibition has been indicated in Fig. 6.

In the antioxidant test, the IC50 of GNPs and BHT were 144 and $201 \mu \mathrm{g} / \mathrm{mL}$, respectively (Table 2).

Studies have shown that the antioxidant features of GNPs green- synthesized by pharmaceutical herbs are more significant than other metal nanoparticles. So far considerable antioxidant activities of GNPs green- synthesized by several pharmaceutic herbs like Gundelia tournefortii L., Allium noeanum Reut. ex Regel, Falcaria vulgaris, Thymus vulgaris, and Camellia sinensis have been confirmed [46]. GNPs green- synthesized by pharmaceutic herbs show noticeable antioxidant activities against free radicals formation in the living system $[45,46]$. The GNPs greensynthesized-formulated have important redox activities and have a noticeable role in free radicals dismantle [46].

Forgoing researches have revealed that phenolic and flavonoids compounds appended to the metallic nanoparticles have important antioxidant activities [46].

As was mention in the introduction Nigella sativa contains antioxidant compounds including thymoquinone, dithymoquinone, t-anethol, thymohydroquinone, dihydroxy-28-methy-lolean-12enoate, p-cymene, 4-terpineol, $\alpha$-pinene, thymol, carvacrol, thymol, sesquiterpene longifolene, 
nigellicine, nigellicimineN-oxide, nigellicimine, nigellidine, alpha-hederin, saponin, citronellol, carvone, limonene, carotene, vitamin A, avenasterol-5-ene, oleic acid, dihomolinoleic acid, eicodadienoic acid, $\alpha$-sitosterol, linoleic acid, campesterol, saponin, stigma-5, $\beta$-amyrin, obtusifoliol, $\beta$-amyrin, cycloartenol, aliphatic alcohol, terpenoids, melanthin, tannin, tirucallol, nigellone gramisterol, $\quad 3-\mathrm{O}$-[ $\beta$-D-xylopyranosyl-( $1 \rightarrow 2)$ - $\alpha$-L-rhamnopyranosyl-( $1 \rightarrow 2)-\beta$-Dglucopyranosyl]-11-methoxy-16, citrostadienol, cycloart-23-methyl-7, Ns-D1, Ns-D2. It seems that the anti-human ovarian cancer effect of recent nanoparticles is due to their antioxidant compounds. Because tumor progression is so closely linked to inflammation and oxidative stress, a compound with anti-inflammatory or antioxidant properties can be an anticarcinogenic agent $[54,55]$.

Many nanoparticles have pharmacological and biochemical properties, including antioxidant and anti-inflammatory properties, which appear to be involved in anticarcinogenic and antimutagenic activities $[55,56]$. Today, nanoparticles synthesized by biological methods play a vital role in treating many diseases, including cancer [55-57]. Nanoparticles synthesized by biological methods are no longer the only ones in traditional medicine, in addition, they have been able to adopt an industrial line of natural products for treating various cancers. Various cell lines from cancers of the prostate, ovary, lung, liver, and pancreas have been treated with metallic nanoparticles [56$58]$. 


\section{Conclusion}

In this research, the GNPs were attained from the reaction between $\mathrm{HAuCl}_{4}$ and Nigella sativa seed aqueous extract in vitro condition. TEM, FE-SEM, UV-Vis, and FT-IR methods were utilized to evaluate nanoparticle characteristics. The results of these techniques revealed that GNPs had been synthesized in the best way. Base on the FT-IR spectrum the presence of a great number of antioxidant compounds produced appropriate conditions for the reduction of gold. In the TEM technique, the mean size of gold nanoparticles (GNPs) was assessed to be $17.5 \mathrm{~nm}$, which is favorable. The GNPs showed the best antioxidant activities against DPPH. In the antioxidant test, the IC50 of GNPs and BHT were 144 and $201 \mu \mathrm{g} / \mathrm{mL}$, respectively. GNPs had appropriate antiovarian cancer activities dose-dependently against SK-OV-3, SW-626, and PA-1 cell lines without any cytotoxicity on the normal cell line (HUVEC). The IC50 of GNPs were 249, 361, and 433 $\mu \mathrm{g} / \mathrm{mL}$ against PA-1, SW-626, and SK-OV-3 cell lines, respectively. After clinical study GNPs containing Nigella sativa seed aqueous extract can be utilized as an efficient drug in the treatment of ovarian cancer in humans.

\section{Conflict of Interest}

The authors declare that there is no conflict of interest. 


\section{Acknowledgement}

This project was supported by Researchers Supporting Project number (RSP-2021/230) King

Saud University, Riyadh, Saudi Arabia.

\section{References}

[1] Goreja WG. Black seed: nature's miracle remedy. New York, NY 7 Amazing Herbs Press; 2003.

[2] Al-Ali A, Alkhawajah AA, Randhawa MA, Shaikh NA. Oral and intraperitoneal LD50 of thymoquinone, an active principle of Nigella sativa, in mice and rats. J Ayub Med Coll Abbottabad 2008; 20(2): 25-27.

[3] Warrier PK, Nambiar VPK, Ramankutty. Indian medicinal plants-a compendium of 500 species. Chennai: Orient Longman Pvt Ltd; 2004, p. 139-142.

[4] Sharma PC, Yelne MB, Dennis TJ. Database on medicinal plants used in Ayurveda. New Delhi; 2005, p. 420-440.

[5] Al-Bukhari MI. In: Sahi Al-Bukhari, editor. The collection of authentic sayings of prophet mohammad (peace be upon him), division 71 on medicine. 2nd ed. Ankara: Hilal Yayinlari; 1976.

[6] Abel-Salam BK. Immunomodulatory effects of black seeds and garlic on alloxan-induced diabetes in albino rat. Allergol Immunopathol (Madr) 2012; 40(6): 336-340.

[7] Khaled AAS. Gastroprotective effects of Nigella Sativa oil on the formation of stress gastritis in hypothyroidal rats. Int J Physiol Pathophysiol Pharmacol 2009; 1: 143-149.

[9] Assayed ME. Radioprotective effects of black seed (Nigella sativa) oil against hemopoietic damage and immunosuppression in gamma-irradiated rats. Immunopharmacol Immunotoxicol 2010; 32(2): 284-296.

[10] Abdel-Zaher AO, Abdel-Rahman MS, Elwasei FM. Protective effect of Nigella sativa oil against tramadol-induced tolerance and dependence in mice: role of nitric oxide and oxidative stress. Neurotoxicology 2011; 32(6): 725-733.

[11] Boskabady MH, Mohsenpoor N, Takaloo L. Antiasthmatic effect of Nigella sativa in airways of asthmatic patients. Phytomedicine 2010; 17(10): 707-713. 
[12] Goreja WG. Black seed: nature's miracle remedy. New York, NY 7 Amazing Herbs Press; 2003.

[13] Warrier PK, Nambiar VPK, Ramankutty. Indian medicinal plants-a compendium of 500 species. Chennai: Orient Longman Pvt Ltd; 2004, p. 139-142.

[14] Yarnell E, Abascal K. Nigella sativa: holy herb of the Middle East. Altern Compl Therapy 2011; 17(2): 99-105.

[15] Padhye S, Banerjee S, Ahmad A, Mohammad R, Sarkar FH. From here to eternity-the the secret of Pharaohs: Therapeutic potential of black cumin seeds and beyond. Cancer Ther 2008; 6: 495-510.

[16] Nickavar B, Mojab F, Javidnia K, Amoli MA. Chemic al composition of the fixed and volatile oils of Nigella sativa L. from Iran. Z Naturforsch C 2003; 58(9-10): 629-631.

[17] Morikawa T, Xu F, Ninomiya K, Matsuda H, Yoshikawa M. N.mines A3, A4, A5 and C, new dolabellane-type diterpene alkaloids with lipid metabolism-promoting activities from the Egyptian medicinal food black cumin. Chem Pharm Bull 2004; 52(4): 494-497.

[18] Morikawa T, Xu F, Kashima Y, Matsuda H, Ninomiya K, Oshikawa M. Noveldolabellanetype diterpene alkaloids with lipid metabolism promoting activities from the seeds of Nigella sativa. Org Lett 2004; 6(6): 869-872.

[19] Ali Z, Ferreira D, Carvalho P, Avery MA, Khan IA. Nigellidine-4-O-sulfite, the first sulfated indazole-type alkaloid from the seeds of Nigella sativa. J Nat Prod 2008; 71(6): 1111-1112.

[20] Mehta BK, Pandit V, Gupta M. New principles from seeds of Nigella sativa. Nat Prod Res. 2009; 23(2): 138-48.

[21] Noor NA, Aboul Ezz HS, Faraag AR, Khadrawy YA. Evaluation of the antiepileptic effect of curcumin and Nigella sativa oil in the pilocarpine model of epilepsy in comparison with valproate.Epilepsy Behav 2012; 24(2): 199-206.

[22] Khader M, Bresgen N, and Eckl PM. In vitro toxicological properties of thymoquinone. Food Chem Toxicol 2009; 47(1): 129-133.

[23] Agarwal C, Narula A, Vyas DK, Jacob D. Effect of seeds of kalaunji on fertility and sialic acid content of the reproductive organs of male rat. Geo Bios 1990; 17: 269-272.

[24] Aqel M, Shaheen R. Effects of the volatile oil of Nigella sativa seeds on the uterine smooth muscle of rat and guinea pig. J Ethnopharm 1996; 52(1): 23-26.

[25] Akhtar M, Maikiyo AM, Khanam R, Mujeeb M, Aqil M, Najmi AK. Ameliorating effects of two extracts of Nigella sativa in the middle cerebral artery occluded rat. J Pharm Bioallied Sci 2012 4(1): 70-75.

[26] Gokce A, Oktar S, Koc A, Yonden Z. Protective effects of thymoquinone against methotrexate-induced testicular injury. Hum Exp Toxicol 2011; 30(8): 897-903.

[27] Hadjzadeh MA, Keshavarzi Z, Yazdi TSA, Ghasem SM, Rajaei Z, Khajavi Rad A. Effect of alcoholic extract of Nigella sativa on cisplatin-induced toxicity in rat. Iran J Kidney Dis 2012; 6(2): 99-104. 
[28] Zafeer MF, Waseem M, Chaudhary S, Parvez S. Cadmium-induced hepatotoxicity and its abrogation by thymoquinone. J Biochem Mol Toxicol 2012; 26(5): 199-205.

[29] Lei X, Liu M, Yang Z, Ji M, Guo X, Dong W. Thymoquinone Prevents and ameliorates dextran sulfate sodium-induced colitis in mice. Dig Dis Sci 2012; 57(9): 2296-2303.

[30] Nemmar A, Al-Salam S, Zia S, Marzouqi F, Al-Dhaheri A, Subramaniyan D, et al. Contrasting actions of diesel exhaust particles on the pulmonary and cardiovascular systems and the effects of thymoquinone. Br J Pharmacol 2011; 164(7): 1871-1882.

[31] Duncker SC, Philippe D, Martin-Paschoud C, Moser M, Mercenier A, Nutten S. Nigella sativa (Black Cumin) seed extract alleviates symptoms of allergic diarrhea in mice, involving opioid receptors. PLoS One 2012; 7(6): e39841.

[32] Nikakhlagh S, Rahim F, Aryani FH, Syahpoush A, Brougerdnya MG, Saki N. Herbal treatment of allergic rhinitis: the use of Nigella sativa. Am J Otolaryngol 2011; 32(5): 402-407.

[33] Lei X, Lv X, Liu M, Yang Z, Ji M, Guo X, et al. Thymoquinone inhibits growth and augments 5-fluorouracil-induced apoptosis in gastric cancer cells both in vitro and in vivo. Biochem Biophys Res Commun 2012; 417(2): 864-868.

[34] Benhaddou-Andaloussi A, Martineau L, Vuong T, Meddah B, Madiraju P, Settaf A, et al. The in vivo antidiabetic activity of Nigella sativa is mediated through activation of the AMPK pathway and increased muscle glut4 content. Evid Based Complement Alternat Med 2011; 2011: 538671.

[35] El Shenawy NS, Soliman MF, Reyad SI. The effect of antioxidant properties of aqueous garlic extract and Nigella sativa as anti-schistosomiasis agents in mice. Rev Inst Med Trop Sao Paulo 2008; 50(1): 29-36.

[36] Harzallah HJ, Grayaa R, Kharoubi W, Maaloul A, Hammami M, Mahjoub T. Thymoquinone, the Nigella sativa bioactive compound, prevents circulatory oxidative stress caused by 1,2dimethylhydrazine in erythrocyte during colon postinitiation

[37] Rogozhin EA, Oshchepkova YI, Odintsova TI, Khadeeva NV, Veshkurova ON, Egorov TA, et al. Novel antifungal defensins from Nigella sativa L. seeds. Plant Physiol Biochem 2011; 49(2): 131-137.

[38] Ahmad, A., Husain, A., Mujeeb, M., Khan, S. A., Najmi, A. K., Siddique, N. A., Damanhouri, Z. A., \& Anwar, F. (2013). A review on therapeutic potential of Nigella sativa: A miracle herb. Asian Pacific Journal of Tropical Biomedicine, 3(5), 337-352. https://doi.org/10.1016/s2221$\underline{1691(13) 60075-1}$

[39] GBD 2015 Disease and Injury Incidence and Prevalence Collaborators. Global, regional, and national incidence, prevalence, and years lived with disability for 310 diseases and injuries, 19902015: a systematic analysis for the Global Burden of Disease Study 2015. Lancet. 2016; 388 : 1545-1602.

[40] GBD 2015 Mortality and Causes of Death Collaborators. Global, regional, and national life expectancy, all-cause mortality, and cause-specific mortality for 249 causes of death, 1980-2015: a systematic analysis for the Global Burden of Disease Study 2015. Lancet. 2016; 388: 1459-1544. [41] Ebell MH et al. A Systematic Review of Symptoms for the Diagnosis of Ovarian Cancer. Am. J. Prev. Med. 2016; 50: 384-394. 
[42] Grossman DC et al. Screening for Ovarian Cancer: US Preventive Services Task Force Recommendation Statement. JAMA. 2018; 319: 588-594.

[43] Gibson SJ et al. The Application and Outcome of Standard of Care Treatment in Elderly Women With Ovarian Cancer: A Literature Review Over the Last 10 Years. Front. Oncol. 2016; 6: 63.

[44] Mohammadi G, Zangeneh MM, Zangeneh A, et al. Appl Organometal Chem 2020; 34: e5136. DOI:10.1002/aoc.5136.

[45] Jalalvand AR, Zhaleh M, Goorani S, et al. J Photochem Photobiol B 2019; 192: 103-112.

[46] Lu Y, Wan X, Li L, Sun P, Liu G. Synthesis of a reusable composite of graphene and silver nanoparticles for catalytic reduction of 4- nitrophenol and performance as anti-colorectal carcinoma. Journal of Materials Research and Technology. 12 (2021) 1832-1843.

[47] Shaneza A et al. Herbal treatment for the ovarian cancer. SGVU Journal of Pharmaceutical Research \& Education, 2018; 3(2): 325-329.

[48] Arunachalam KD et al. One-step green synthesis and characterization of leaf extract-mediated biocompatible silver and gold nanoparticles from Memecylon umbellatum. Int J Nanomedicine. 2003; 8: 1307-1315.

[49] Tahvilian R, Zangeneh MM, Falahi H, et al. Green synthesis and chemical characterization of copper nanoparticles using Allium saralicum leaves and assessment of their cytotoxicity, antioxidant, antimicrobial, and cutaneous wound healing properties. Appl Organometal Chem. 2019; 33: e5234. DOI: 10.1002/aoc.5234.

[50] You C, Han C, Wang X, et al. The progress of silver nanoparticles in the antibacterial mechanism, clinical application and cytotoxicity, Mol. Biol. Rep. 2012; 39: 9193-9201. https://doi.org/10.1007/s11033-012-1792-8.

[51] Mao BH et al. Mechanisms of silver nanoparticle-induced toxicity and important role of autophagy. Nanotoxicol. 2016; 10: 1021-1040.

[52] Namvar F, Rahman HS, Mohamad R, et al. Cytotoxic effect of magnetic iron oxide nanoparticles synthesized via seaweed aqueous extract. Int J Nanomedicine 2014; 19: 2479-88.

[53] Sankar R, Maheswari R, Karthik S, et al. Anticancer activity of Ficus religiosa engineered copper oxide nanoparticles. Mat Sci Eng C 2014; 44: 234-239.

[54] Katata-Seru L, Moremedi T, Aremu OS, et al. Green synthesis of iron nanoparticles using Moringa oleifera extracts and their applications: Removal of nitrate from water and antibacterial activity against Escherichia coli. J Mol Liq 2018; 256: 296-304.

[55] Sangami S, Manu M. Synthesis of Green Iron Nanoparticles using Laterite and their application as a Fenton-like catalyst for the degradation of herbicide Ametryn in water, Environ. Technol. Innov. 2017; 8: 150-163.

[56] Beheshtkhoo N, Kouhbanani MAJ, Savardashtaki A, et al. Green synthesis of iron oxide nanoparticles by aqueous leaf extract of Daphne mezereum as a novel dye removing material. Appl Phys A 2018; 124: 363-369.

[57] Radini IA, Hasan N, Malik MA, et al. Biosynthesis of iron nanoparticles using Trigonella foenum-graecum seed extract for photocatalytic methyl orange dye degradation and antibacterial applications. J Photochem Photobiol B 2018; 183: 154-163.

[58] Oganesvan G, Galstyan A, Mnatsakanyan V, et al. Phenolic and flavonoid compounds of Ziziphora clinopodioides. Chem Nat 1991; 27: 247-247. 
Table 1. The IC50 of gold nanoparticles in the anti-human ovarian cancer test.

\begin{tabular}{|c|c|c|c|}
\hline & PA-1 & SW-626 & SK-OV-3 \\
\hline IC50 $(\boldsymbol{\mu g} / \mathbf{m L})$ & 249 & 361 & 433 \\
\hline
\end{tabular}


Table 2. The IC50 of gold nanoparticles and BHT in the antioxidant test.

\begin{tabular}{|c|c|c|}
\hline & Gold nanoparticles & BHT \\
\hline IC50 $(\boldsymbol{\mu g} / \mathbf{m L})$ & 144 & 277 \\
\hline
\end{tabular}




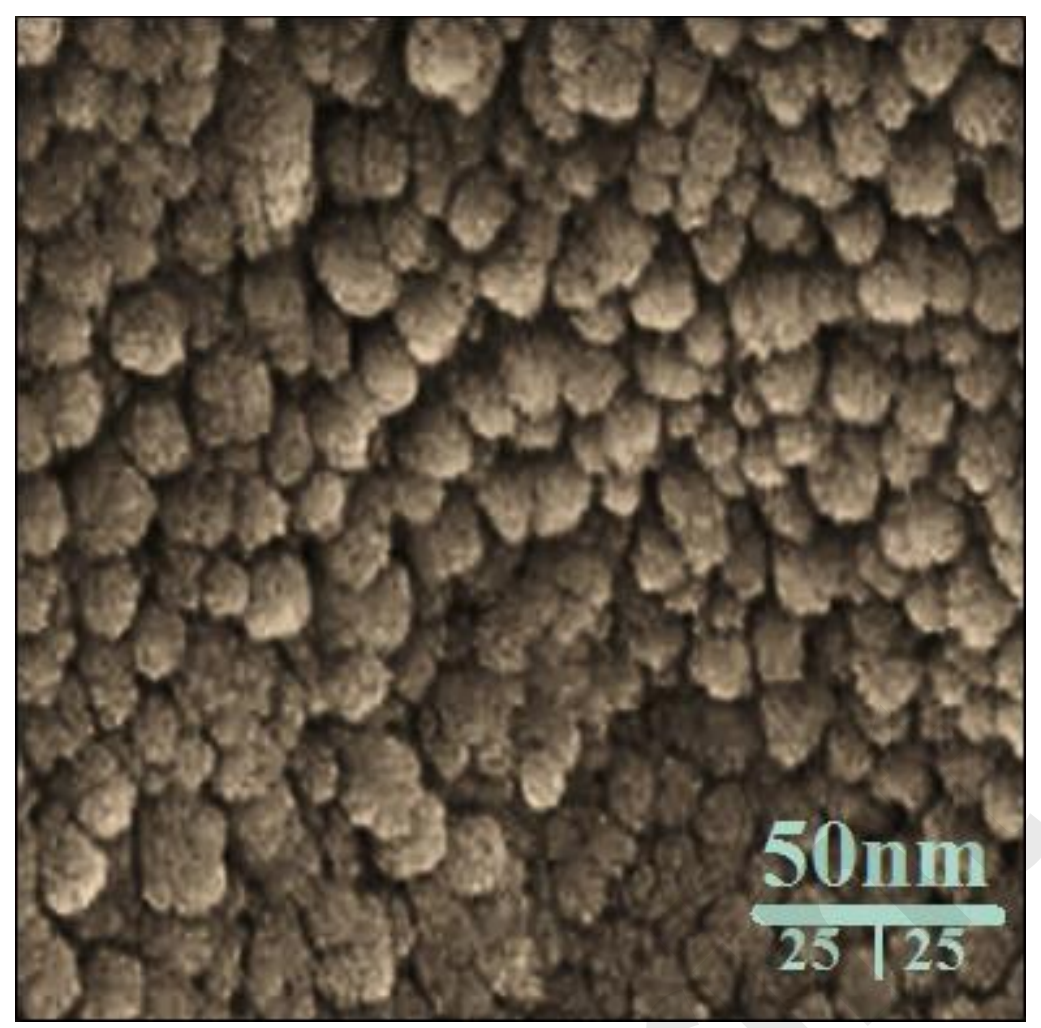

Figure 1. FE-SEM image of GNPs green-synthesized by Nigella sativa seeds. 


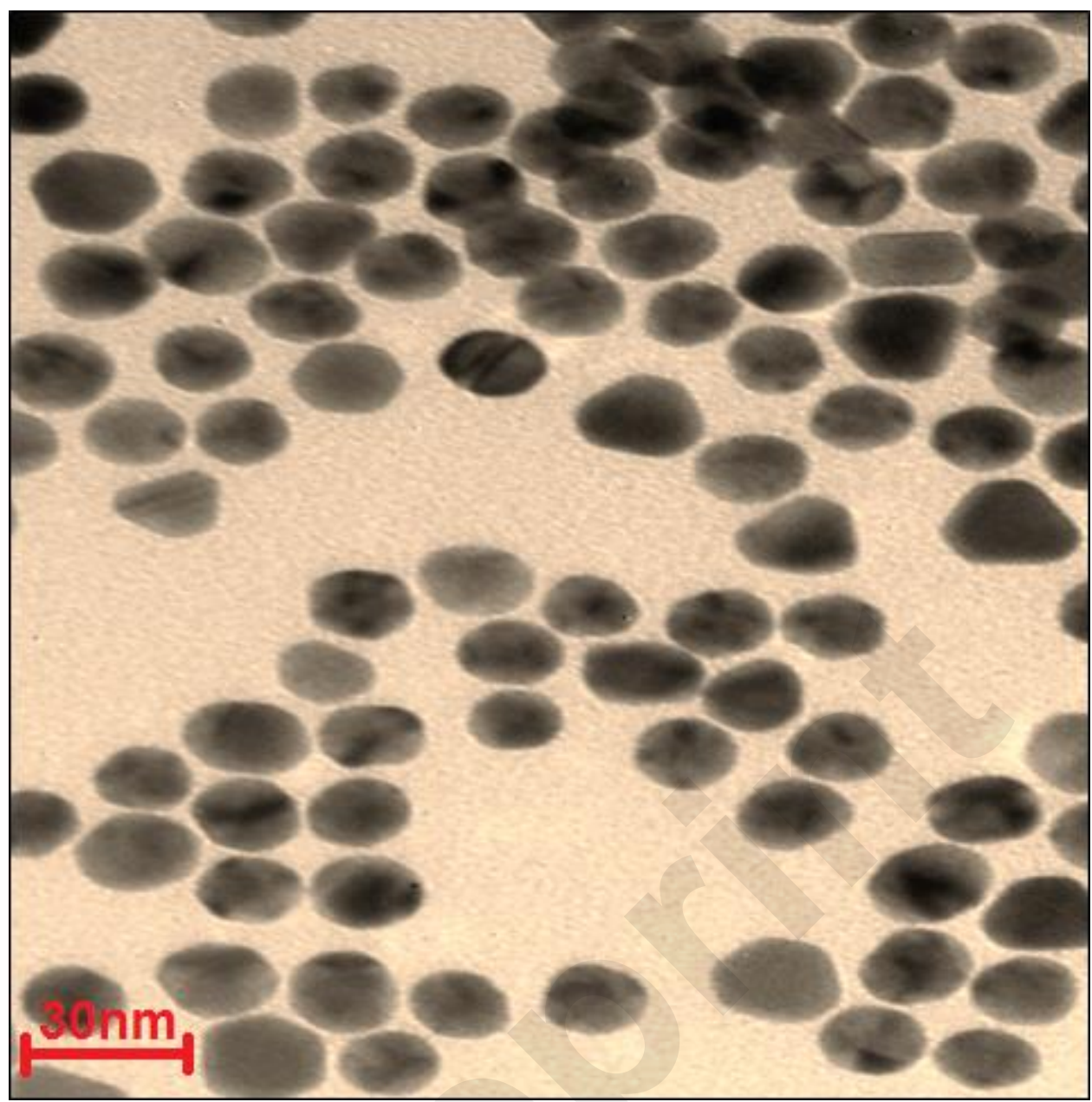

Figure 2. TEM image of Au nanoparticles green-synthesized by Nigella sativa seeds. 


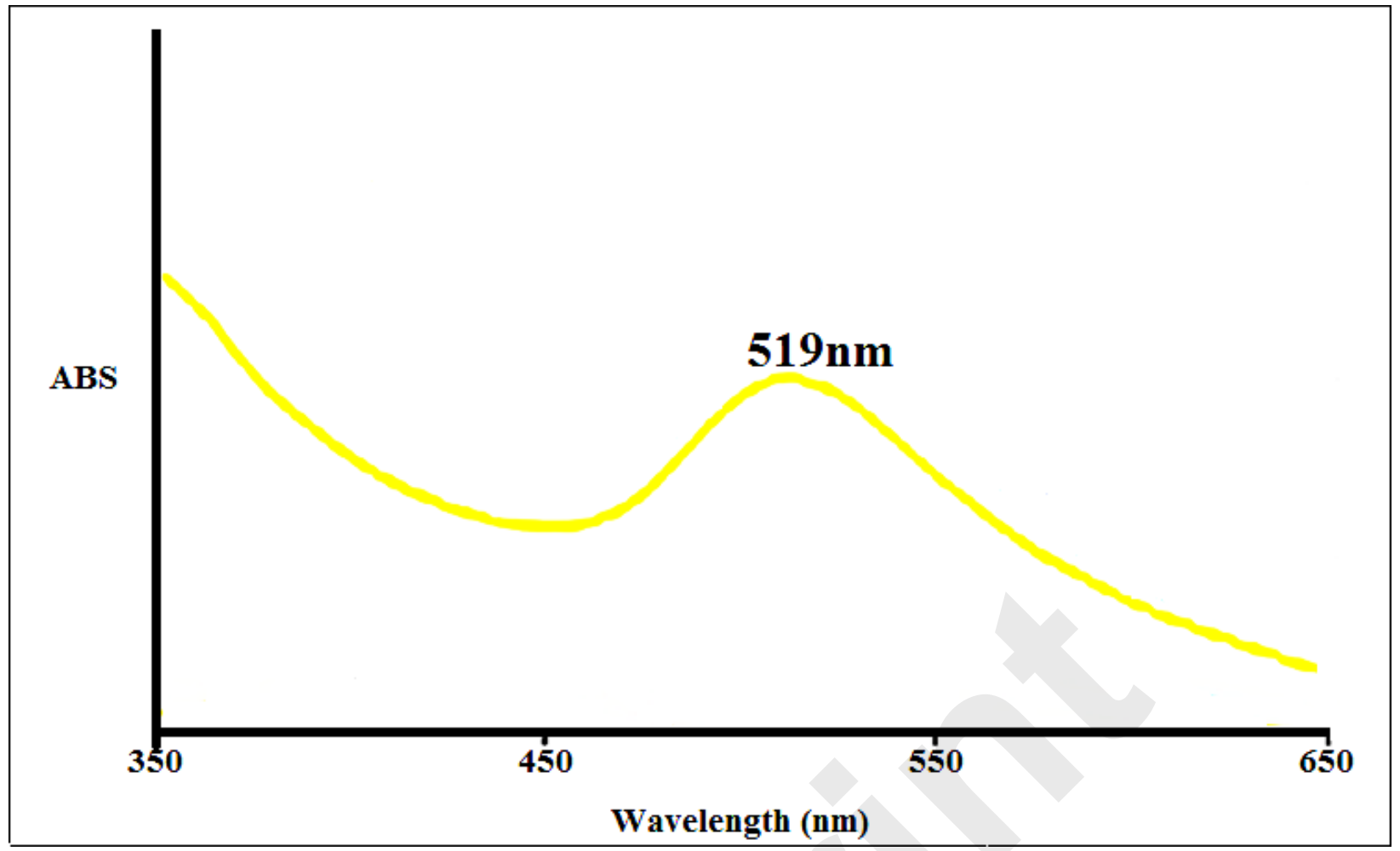

Figure 3. UV-Vis spectra of Au nanoparticles green-synthesized by Nigella sativa seeds. 


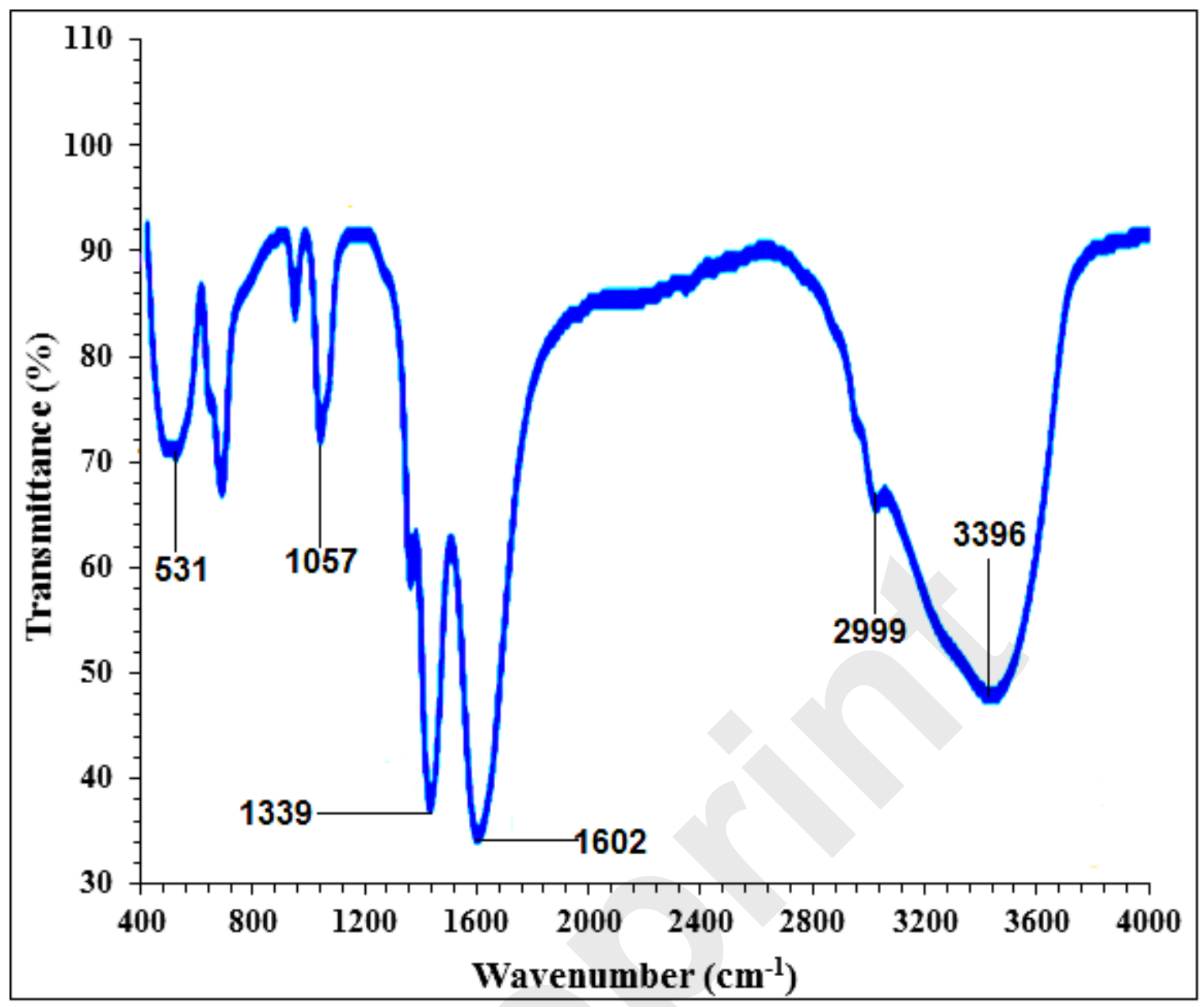

Figure 4. FT-IR analysis of Au nanoparticles green-synthesized by Nigella sativa seeds. 


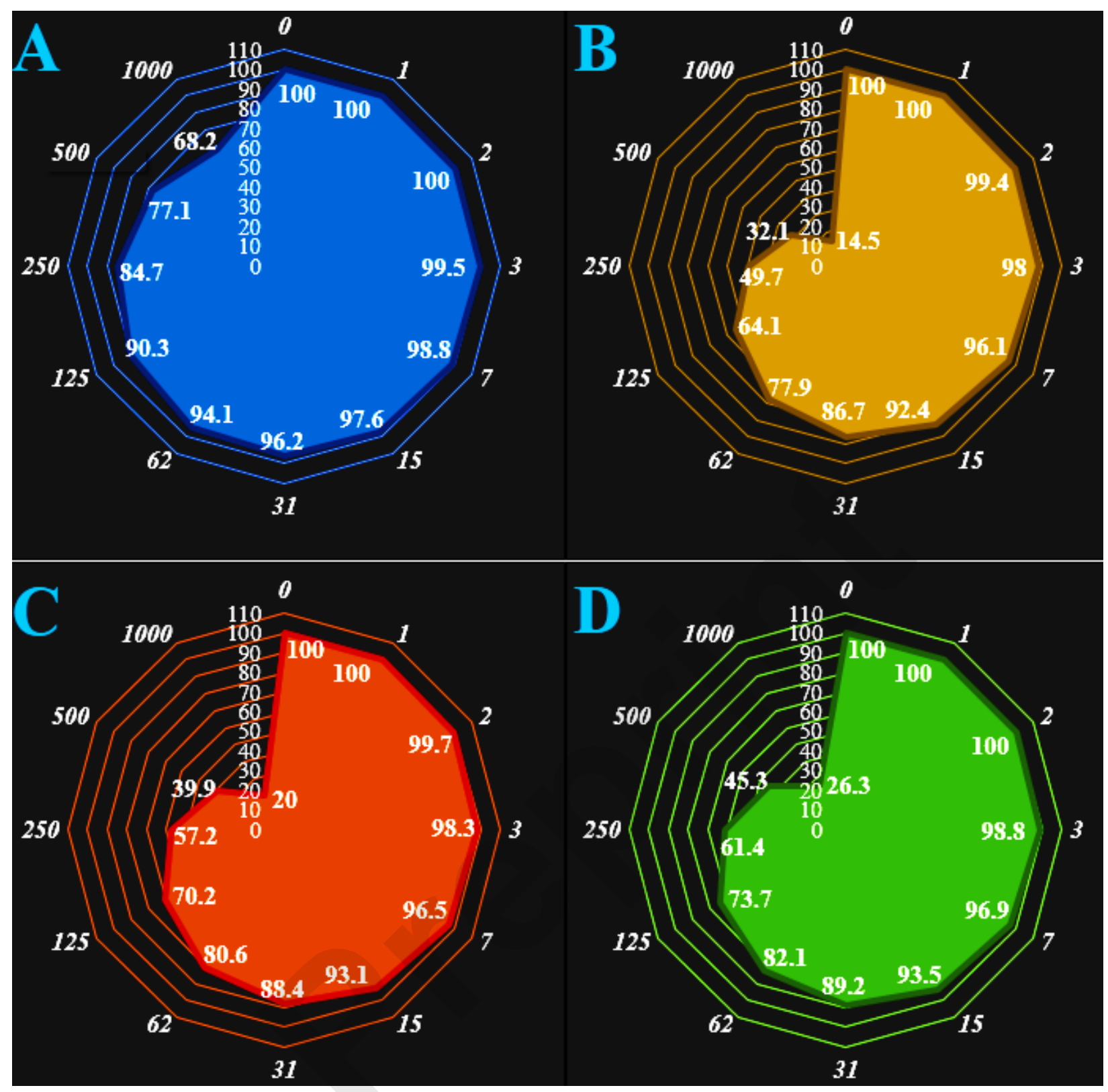

Figure 5. The anti-human ovarian cancer properties (Cell viability (\%)) of gold nanoparticles (Concentrations of 0-1000 $\mu \mathrm{g} / \mathrm{mL}$ ) against normal (HUVEC: A) and human ovarian cancer (PA-1 (A), SW-626 (B), and SK-OV-3 (C)) cell lines.

The numbers indicate the percents of cell viability in the concentrations of $0-1000 \mu \mathrm{g} / \mathrm{mL}$ of gold nanoparticles against several human ovarian cancer cell lines. 


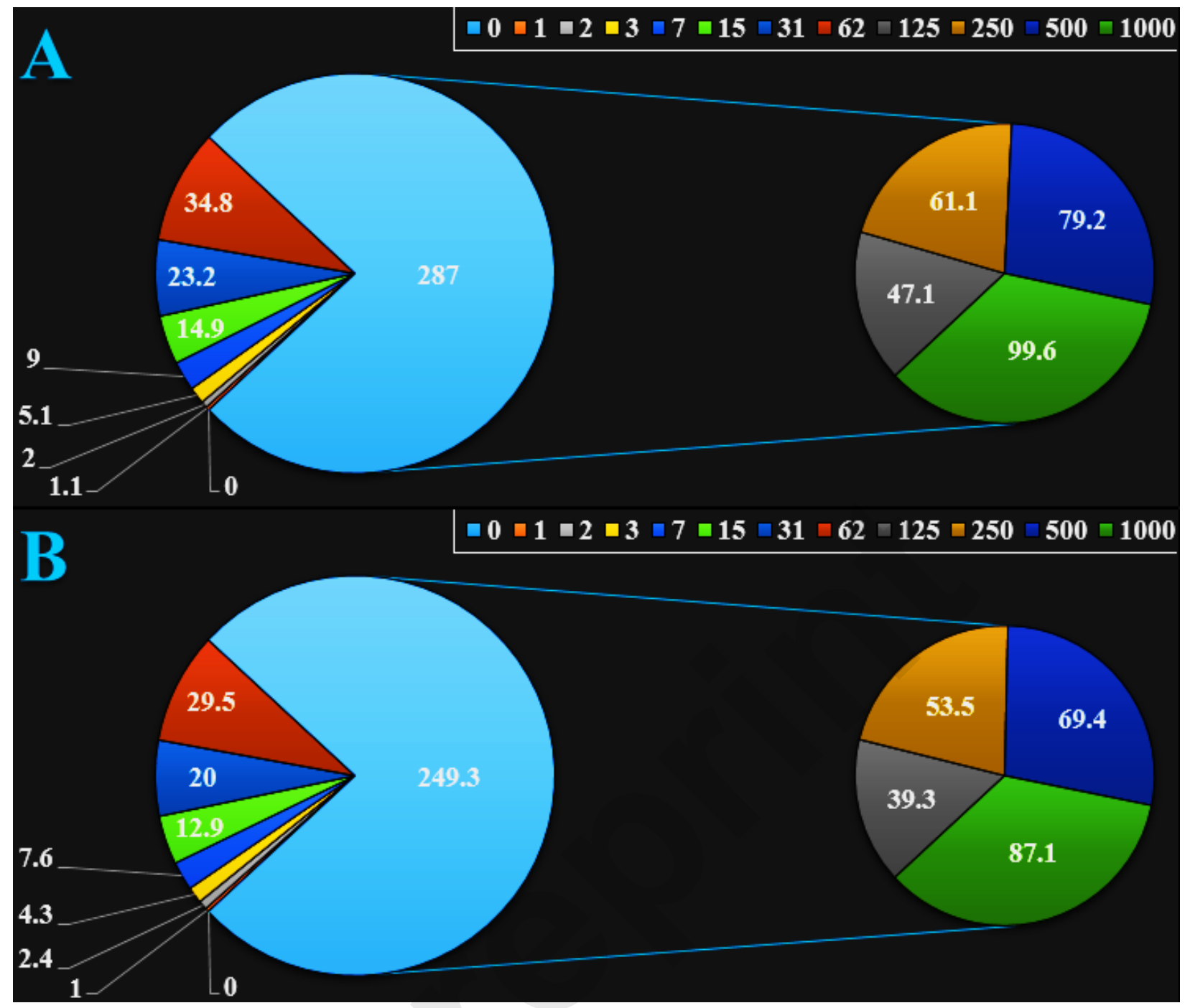

Figure 6. The antioxidant properties of gold nanoparticles (A) and BHT (B) against DPPH.

The numbers indicate the percents of free radical (DPPH) inhibition in the concentrations of $0-1000 \mu \mathrm{g} / \mathrm{mL}$ of gold nanoparticles (A) and BHT (B). 